\title{
Realization of Cross-platform Video and Audio Transmission Technology Based on HTML5
}

\author{
Kai Liang ${ }^{1, a}$, Sanxing $\mathrm{CaO}^{2, \mathrm{~b}}$ \\ ${ }^{1}$ School of Communication University of China, Beijing 100024, China; \\ ${ }^{2}$ School of Communication University of China, Beijing 100024, China; \\ aliangkai_cuc@163.com, bcaosx_cuc@126.com
}

Keywords: HTML5; Video tags; UDP transport protocol; PhoneGap

\begin{abstract}
The current transmission and playback of the streaming media in the WEB browser are mainly depend on the use of the Flash plug-in. And different platforms you need to install the plug-in are often different, that bring a very bad user experience. Combined with UDP transport protocol of streaming media, the design of audio and video players is realized by HTML5 technology in this paper. And create a custom control for HTML5 audio and video with JavaScript MediaAPI, while PhoneGap was used for encapsulation, so as to achieve the cross-platform, cross-terminal application.
\end{abstract}

\section{Introduction}

With the rapid development of mobile Internet, the entrance to Internet such as the browser and APP will become a key to developer. A significant feature of the mobile Internet era is required to cross the terminal, cross the platform, and the development of application can be applied on different platforms and operating systems, the emergence of HTML 5 technology makes it more convenient. Broadly, HTML5 is the general designation of HTML5, CSS3 and Javascript 2.0, because as to the current Internet development, the three are inseparable. The original purpose of HTML5 standards is to reduce the dependence on all kinds of plug-in, while enriching the browser side of the application. Compared with HTML4, HTML5 play media files directly to the built-in support, that it can use the new explicit video and audio tags to handle video and audio data, make the past need to rely on plug-ins in order to support music and video, and now with the support of HTML5 browsers can be a direct coding and decoding for playback. And HTML 5 technology can also be used as a research foundation of streaming media from a Web server to the PC/mobile terminal transmission, it provides a new way of thinking for replacing the traditional streaming media transmission mode and optimizing the audio and video transmission scheme.

\section{Research on supporting technology of HTML5 audio and video}

HTML5 multimedia related tags. HTML 5 involves $<$ video... / > and $<$ audio... / > tags to mark both web video and audio, and does not need to use the plug-in. In addition, HTML5 also provides multimedia player-related attributes, such as video tags contain controls, preload, loop and other property, and an internal used label <source $>$. Audio tag attributes is also similar, which gives developers a free design space. Like other scripts, audio and video elements will trigger a number of events, developers can bind the listener in JavaScript, and call a method according to need or change the properties to control audio and video playback.

The major browsers support for video and audio tags. Due to the historical origin of video encoder, the video format is different from various browsers, there is no video format compatible with all browsers, the only solution is to convert the video to a variety of formats. But with HTML 5 technology standards, most browsers have support for video tag. Video element supports three kinds of video format, namely Ogg, MPEG4, and WebM. 
Table 1, Browsers which support video tags

\begin{tabular}{cccccc}
\hline Format & IE & Firefox & Opera & Chrome & Safari \\
\hline Ogg & No & $3.5^{+}$ & $10.5+$ & $5.0+$ & No \\
MPEG 4 & $9.0^{+}$ & No & No & $5.0+$ & $3.0+$ \\
WebM & No & $4.0+$ & $10.6+$ & $6.0+$ & No \\
\hline
\end{tabular}

From the above table it can be seen that not all browsers are simultaneously supporting video elements of all the three video formats. Although audio elements support three kinds of audio formats, Ogg Vorbis, mp3 and wav, it also have not been unified on all browsers. In this paper, we will put forward a method of cross-browser compatible method to achieve different browser can automatically obtain the corresponding support audio and video format for playback, and the same applies to the mobile terminal browser, which is the basis to achieve cross-platform play.

The selection of the UDP transport protocol. Since this article studies the audio, video and other multimedia data transmission, transfer speed and transmission performance is the first factor to be considered, so the UDP protocol is more suitable for transmission layer protocol selection. If you are experiencing network congestion or network latency, UDP protocol can use the lower standard and high speed to transfer data packets, and as the same layer of the TCP transport protocol have higher request for transmission bandwidth, obviously do not have the conditions of the complex network transmission.

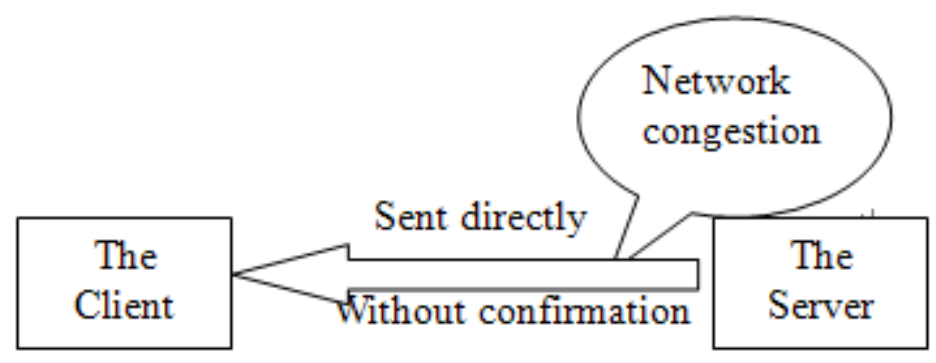

Fig.1 UDP transport protocol

\section{The design and optimization of HTML5 video and audio player}

The design of the HTML5 audio player. HTML5 Audio tag is used to implement the design of the Audio player, which involves play/pause button, broadcast schedule control, play time,audio time, volume button. Right click to support video and audio file save , Playback speed, audio URL and other information. Some of the key code is as follows:

<audio controls="controls"> < source src="The rose.ogg" type="audio/ogg">

$<$ source src="The rose.mp3" type="audio/mpeg">Your browser does not support the audio tag. $</$ audio $>$

The design of the The HTML5 video player. Based on the HTML 5 Video tags, and calls the head tags within the CSS file to achieve the front-end interface design of HTML 5 player. At the same time, the reference JavaScript files is used to realize the custom control of the video player.

$<$ !Doctype html $><$ html $><$ head $>$

$<$ link href="style.css" rel="stylesheet" type = "text/CSS" $><$ script src="style.js" $></$ script $>$

$<$ video width="480" height="320" controls="controls" $><$ source src="movie.ogg"

type="video/ogg" $><$ source src= "movie.mp4" type="video/mp4" $><$ source src="moive.webm" type="video/webm" />Your browser does not support the video tag. $</$ video $>$

Among them, the file of style.js mainly contains MediaAPI JavaScript to control video object, such as fast forward/back, adjusting the size and replaying the control. The following code is to realize the function of re-play:

<script type = "text/javascript” $>$ var myMoive = docment.getElementById (“moive”); 
function restartMoive ()$\{$ myMoive.current.Time $=0 ;\}$

$<$ script $>$

Optimization of HTML5 video player. As mentioned above, not all browsers support both audio and video format of HTML 5, but it can automatically grab corresponding supported video format through source tags. For the older version of browsers, we can replace the prompt content when the browsers do not support with the traditional flash. So that when the browser is not compatible with the video tag, it will show that the flash version of the video, which makes the player has extensibility and openness on the choice of video type.

<object width="480" height="240" type="application/x-shockwave-flash" data="fallback.swf"> $<$ param name="movie" value="fallback.swf" />

$<$ param name="flashvars" value="autostart=true\&amp; file=moive.flv"/>

$<$ object $>$

\section{Implementation of the cross-platform transmission process of HTML 5 video and audio}

The design of system function and structure.

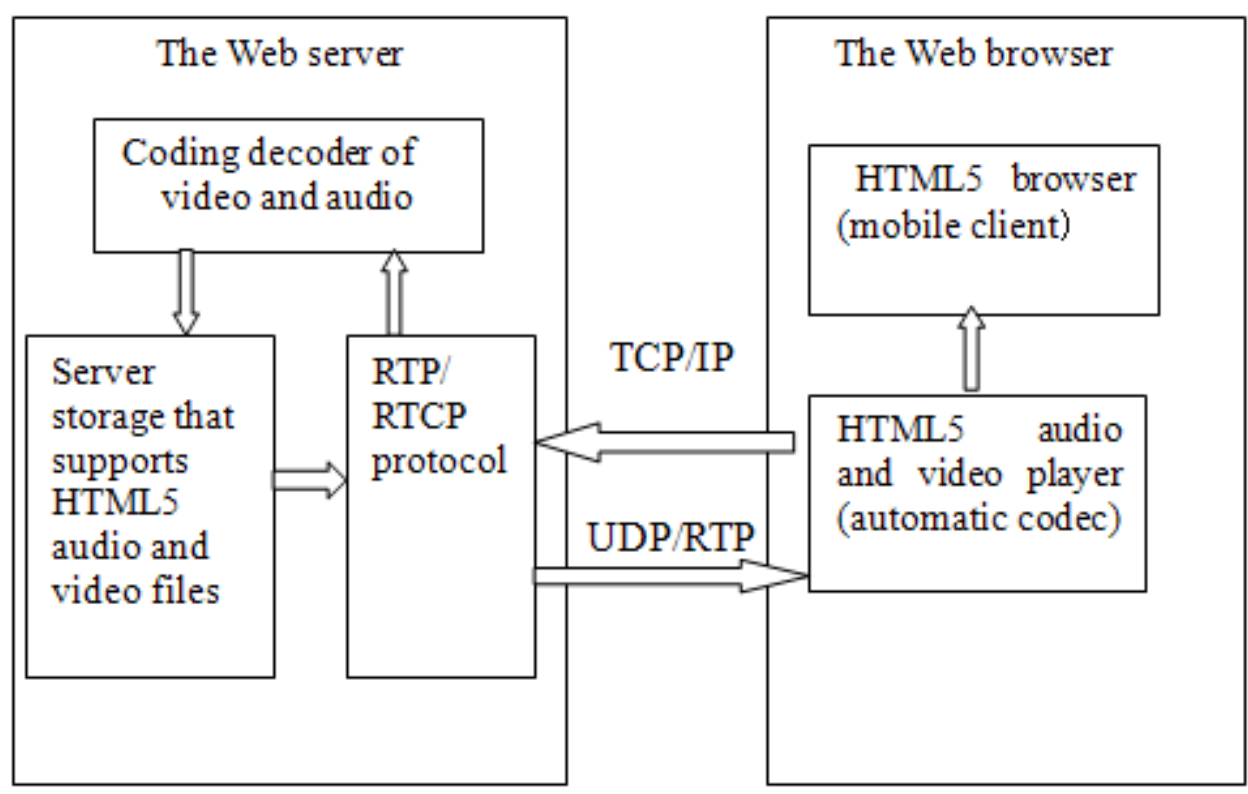

Fig.2 System function and structure

From above, we can see that the system is composed of Web server, transmission protocol, and Web browser. Working principle is as follows, the Web browser requests to a web server to load video stream, the Web server decoding the audio and video files after receives the request, then packaging audio and video stream with the RTP protocol, and finally run the UDP/RTP protocol to send out the packaged video. At the web browser client, it will receive video and audio reassembly, codec and then playback on the HTML5 audio and video player.

The realization of the cross-platform transfer. PhoneGap can support for the language of the native API calls, including HTML5. Therefore, in this paper, PhoneGap is used to encapsulate the HTML5 audio and video players, so as to realize the cross-platform transmission of the mobile terminal, that is also to develop a WebApp. It packaged HTML5 + CSS + JavaScript files into a similar "image file", and the implementation is explained directly by the browser when running, in order to achieve a development for multi-platform compatibility. 


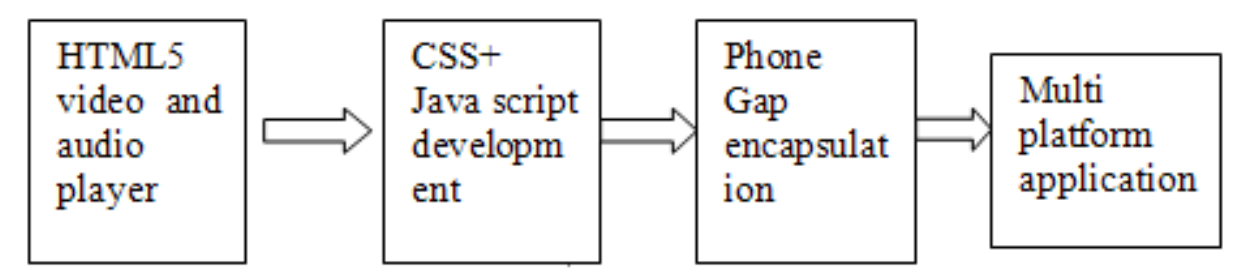

Fig.3 Cross-platform transfer

\section{Summary}

Although the main technology of video transmission is still streaming media technology, with the development of HTML5 technology, more and more web browser manufacturer support HTML5 standard, it will become an inevitable trend that audio and video players constructed by HTML5 instead of the traditional streaming media transmission. In this paper, with the help of HTML5's video and audio tags, we achieve the design of audio and video player, and integrate the traditional streaming media with HTML5 to achieve the cross platform transmission of audio and video. It also realize the function of HTML5 online video and audio at the same time, which has a certain reference effect for the development of Internet video and audio industry.

\section{References}

[1] Bruce Lawson, Vadim Makeev. HTML5 Embed Video Into Your Pages.[J]. net.July 2010,P 80-83

[2] Lourdes Moreno, Paloma Martinez, Ana Zglesias,and Maria Gonzalez. HTML5 Support for an Accessible User-Video-Interaction on the Web.[J]. DFIP International Federation for Infotxnation Processing,2011,P 535-539

[3] Matt Wiggins.createb turning a Video into a puzzle.[J]. .net,September 2011,P 120

[4] John Dyer. Practiacl Cross-Browser HTML5 Audio And Video.[J].msdn magazine .2012: 46-52.

[5] Mohammad S Kayyali. Web APIs on the march.[J]..net.summer 2012.P 20

[6] PhoneGap Build is out of Beta [EB/OL].http:// www.phonegap.com/ 